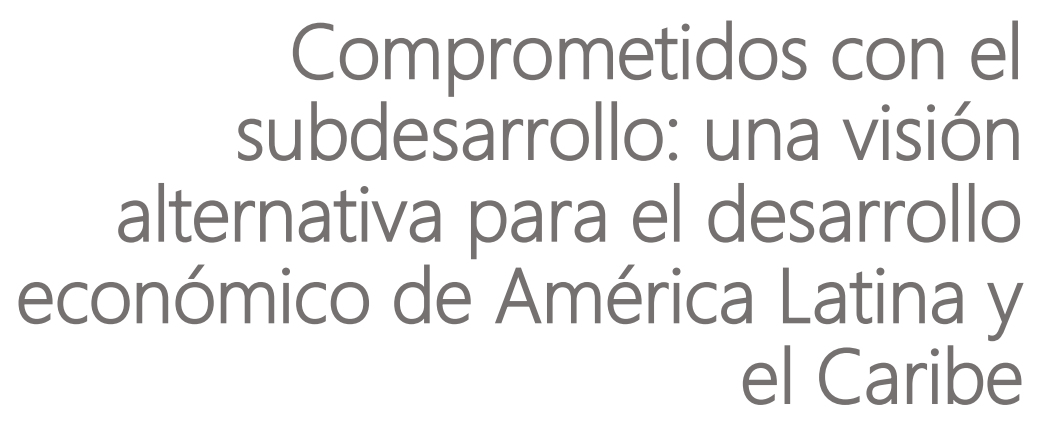

Oscar Esteban Morillo Martínez Universidad Nacional de Colombia Colombia 


\section{Comprometidos con el subdesarrollo: una visión alternativa para el desarrollo económico de América Latina y el Caribe}

Oscar Esteban Morillo Martínez

Universidad Nacional de Colombia - Colombia

\section{RESUMEN}

El desarrollo en América Latina y el Caribe debe pensarse como un problema de restricción externa, donde se han impuesto discursos que impiden cualquier proceso de convergencia con las economías desarrolladas. Por esto, se hace necesario cuestionar los fundamentos teóricos de tales discursos, demostrando que, en su mayoría, han sido controvertidos y declarados inconsistentes; transformando tales posturas en ideológicas y nunca coherentes. Así pues, en este artículo se busca afrontar los desafíos del desarrollo que enfrenta la región a partir de un análisis teórico alternativo que nos acerque a una concepción global de los conflictos socio-económicos del desarrollo y nos permita sostener postulados normativos para contribuir al debate por el desarrollo de nuestras naciones, abogando siempre por políticas de industrialización y de crecimiento vía demanda efectiva.

Palabras clave: Desarrollo económico; Demanda efectiva; Finanzas funcionales; Ley de Kaldor.

Autor de correspondencia: Oscar Esteban Morillo Martínez omorillo@unal.edu.co

Editor: Andrés Escobar E. Universidad de Cartagena-Colombia.

Tipología IBN Publindex:

Artículo de Revisión
Copyright: (C) 2021. Morillo Martínez, O. Este es un artículo de acceso abierto, distribuido bajo los términos de la licencia https://creativecommons.org/licenses/bync-sa/4.0/ la cual permite el uso sin restricciones, distribución y reproducción en cualquier medio, siempre y cuando que el original, el autor y la fuente sean acreditados.

\section{Committed to underdevelopment: an alternative vision for economic development in Latin America and the Caribbean}

\section{ABSTRACT}

Development in Latin America and the Caribbean should be thought of as a problem of external restriction, where discourses have been imposed that impede any process of convergence with developed economies. For this reason, it is necessary to question the theoretical foundations of such discourses, showing that, for the most part, they have been controversial and declared inconsistent; transforming such positions into incoherent ideologies. Thus, this article seeks to present the development challenges facing the region based on an alternative theoretical analysis that brings us closer to a global conception of the socio-economic conflicts of development and allows us to support normative postulates to contribute to the debate on the development of our nations. We always advocate for industrialization and growth policies via effective demand.

Keywords: Economic development; Effective demand; Functional finance; Kaldor's law.

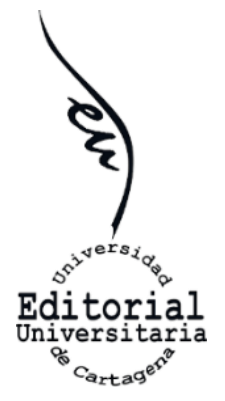




\section{INTRODUCCIÓN}

Pensar en América Latina y el Caribe implica pensar en un problema de dependencia estructural, donde nos hemos configurado como periferia global en el sistema mundo contemporáneo y donde se han abandonado todos los intentos de industrialización a cambio de una paupérrima inserción a las cadenas globales de valor, por lo general, a través de profundizar la primarización de nuestras economías; generando así profundas lógicas de violencia que reproducen las desigualdades sistémicas de la región.

El desarrollo será entendido como un proceso de progreso económico y social. Donde lo fundamental no es el crecimiento del producto sino el mejoramiento de las condiciones materiales y sociales de la población. Esta visión sostiene que de nada sirve un fuerte crecimiento del PIB si esto no se refleja en una reducción de la desigualdad y mejoramiento de indicadores como el empleo o la distribución funcional del ingreso. En últimas, el desarrollo económico tiene que ver con la sustentabilidad de un determinado régimen de acumulación, y cómo esta sustentabilidad debe proyectarse en una serie de políticas y determinaciones económicas tomadas por los diferentes gobiernos nacionales.

Así las cosas, el problema del desarrollo en América Latina y el Caribe puede resumirse, guardadas proporciones, en un problema de subdesarrollo de la estructura productiva y del desarrollo tecnológico de los diferentes países. Como regla general de nuestras economías, se importa tecnología y bienes de capital desde el centro global (Prebisch, 1983) para dar paso a la producción especializada en sectores primarios que profundizan las lógicas desiguales y especulativas de acumulación de la región.

Esta dependencia tecnológica (Prebisch, 1983) implica, a su vez, que cualquier intento de transformación productiva (entiéndase procesos de industrialización por sustitución de importaciones) chocará con una restricción externa de divisas internacionales, es decir, con déficits en cuenta corriente y balanza de pagos que hacen insostenible el crecimiento económico a largo plazo para una economía (Thirlwall, 1979). Dicha restricción se explica por las relaciones técnicas de producción existentes en las diferentes economías de la región que, dada su configuración insumo-producto, hace que aquellos sectores básicos ${ }^{1}$ inexistentes en la matriz productiva doméstica se reemplacen vía importaciones, lo cual, evidentemente, requiere divisas internacionales; en especial del dólar, moneda que funciona como unidad de cuenta internacional.

Para analizar esta situación es necesario realizar un abordaje, en primera instancia, teórico; el cual ha sido abandonado por la disciplina en su afán de obtener resultados empíricos sin ninguna trascendencia teórica y ostentando una supuesta neutralidad técnica. Se hace relevante la teoría debido a que las diferentes visiones de mundo que se tenga configurarán nuestro accionar con el mismo; esta performatividad de las ideas (Austin, 2016) implicará que para modificar los resultados económicos de nuestra región se debe partir de un marco teórico alternativo, que dé pie a formulaciones de política pública que garanticen el crecimiento económico estable y sostenible sin deteriorar las condiciones materiales de la sociedad².

\footnotetext{
${ }^{1}$ La referencia a bienes básicos sigue el sentido propuesto por Sraffa (1960).

${ }^{2}$ Los economistas tienden a rehuir a las discusiones epistemológicas, siendo que su acercamiento más preciso se remite a los hechos estilizados (Kaldor, 1957), enfoque que adoptaremos en este documento.
} 
En este sentido, el presente artículo busca controvertir los principales postulados teóricos del mainstream económico, entendiendo que éstos fijan las formulaciones de política pública más comunes en toda la región, para promover una visión alternativa y heterodoxa sustentada en los trabajos de Sraffa (1960), Keynes (1965), Kaldor (1957, 1966, 1972 y 1975) y Prebisch (1983) que nos acerque a recomendaciones y soluciones estructurales que signifiquen una superación de la profunda dependencia y desigualdad de la región.

\section{EL DISCURSO}

Aunque algunos economistas pretendan reducir la crítica de Cambridge a una discusión por la validez en la medición del capital, en realidad sólo tocan la superficie empírica de la discusión. El resultado más importante de las controversias de Cambridge se refiere a la imposibilidad de considerar el capital como un factor de producción ${ }^{3}$ (Sraffa, 1960), lo cual significa que deja de existir el "efecto sustitución" haciendo imposible el ajuste de la economía hacia el pleno empleo (Garegnani, 1978-79).

No obstante, como se mencionó en un primer momento, los economistas han pasado por alto el alcance de estas controversias e incluso han obviado la gran aceptada imposibilidad de la agregación del capital ${ }^{4}$, mostrando una deliberada omisión de las discusiones teóricas que se hace evidente en los manuales de economía que continúan presentando la función de producción neoclásica tradicional, casi siempre, sin mención alguna a los debates del capital. Además, esta omisión se observa en los congresos internacionales, conferencias, publicaciones científicas $y$, de forma catastrófica, en la política económica.

Así las cosas, la ignorancia deliberada del callejón sin salida en que se convirtió la teoría neoclásica ha provocado que, en buena parte de las últimas décadas, los proyectos económicos y las configuraciones políticas regionales y nacionales se mantengan sustancialmente inamovibles. Esto es, que las principales formulaciones de política económica se mantengan estáticas y sean compatibles con el bloque de poder de turno. De forma tal que, los proyectos de desarrollo económico han estado alejados de cualquier intención de transformación productiva y, más bien, se han concentrado en dinamizar su economía y su crecimiento vía devaluaciones (Rapetti et al, 2012), especializaciones productivas (ventajas comparativas) e inserciones en cadenas globales de valor (Bielschowsky, 2009). Esta visión se ata a programas de austeridad y de finanzas "sanas" ${ }^{\prime}$ que, supuestamente, hacen proclive la estabilidad macroeconómica de largo plazo y permiten a las economías de la periferia alcanzar sendas de crecimiento que permitan converger sus niveles de producto con las economías desarrolladas (CEPAL, 2012).

\footnotetext{
${ }^{3}$ Nótese que esta implicación para la teoría de la distribución (no sólo neoclásica) es pasada por alto por una amplia cantidad de economistas que se concentran en la discusión de la medición y formulación de una función de producción agregada, por ejemplo, Blaug (1985).

${ }^{4}$ Los padres de la teoría neoclásica moderna, Samuelson (1966) y Solow (1975), aceptaron la imposibilidad de la medición del capital. Incluso Hayek (1946), eminente economista austriaco, reconoce que no se puede tratar al capital como una sustancia homogénea.

${ }^{5}$ Las finanzas "sanas" surgen del discurso que busca evitar la generación de déficits macroeconómicos, suponiendo que esto genera mayor estabilidad económica en el largo plazo. Esta perspectiva difiere radicalmente de la visión de finanzas funcionales de Lerner (1943 y 1951).
} 
Sin embargo, estas visiones deben ser discutidas en tanto han terminado por promover la inestabilidad y la vulnerabilidad de las economías regionales. Por un lado, gracias al abandono de los proyectos productivos nacionales y, por el otro, por la promoción de los flujos de capital como bases para el crecimiento económico, situación que sumerge en dinámicas de dependencia estructural y financiera (Bonizzi, 2013) a las economías periféricas respecto a los países del centro global (Bortz y Kaltenbrunner, 2018).

En primera instancia, cuando se propone el comercio internacional como fuente primaria de crecimiento y desarrollo económico se asume que, sin importar las diferencias tecnológicas, los salarios suficientemente bajos harán posible la competencia internacional de los países (Krugman, 1991). De esta forma, la postura mainstream que parte de los modelos de comercio ricardianos (Dornbusch et al, 1977) supone la existencia de bienes producidos únicamente con trabajo, lo cual, evidentemente, hace imposible la exclusión del comercio internacional, pues cualquier caída en los salarios hace reducir arbitrariamente los costos de producción relativos al resto del mundo y permite la superación de todo atraso tecnológico, es decir, de productividad.

Sin embargo, siguiendo el trabajo de Crespo et al. (2019) y de Parrinello (2010) se puede evidenciar, desde el marco analítico de un modelo de comercio ricardiano, que la introducción de bienes de capital (así éstos sean entendidos como salarios pagados previamente) y de supuestos de movilidad factorial, hacen plausible la exclusión del comercio internacional al imponer un límite inferior a los costos de producción de la economía particular, esto es, impidiendo que la inserción internacional se logre a través de la disminución de los salarios. Situación que controvierte los principales resultados de la teoría convencional del comercio internacional.

En segunda instancia, la propuesta de apalancar el crecimiento en la devaluación de la moneda nacional para subsanar las diferencias en costos de producción ${ }^{6}$ es una propuesta que sigue las líneas del neoestructuralismo, presente en la CEPAL (Bielschowsky, 2009) y que sostiene que, en el corto plazo, un incremento en la tasa de cambio acelera el crecimiento económico a través de sus efectos expansivos en el empleo y en las exportaciones del sector transable (se asume que es la industria). En el mediano plazo, se incrementará la participación en el ingreso nacional del sector más competitivo y en el largo plazo este incremento en la tasa de ganancia se traducirá en un régimen de crecimiento guiado por las ganancias (Dvoskin et al, 2018).

La aplicación de esta visión desconoce los efectos contractivos que implica la devaluación (Krugman y Taylor, 1978), asumiendo que serán pasivamente absorbidos por las exportaciones (Frenkel y Ros, 2006). A la vez, que está sustentada en varios supuestos muy restrictivos, entre ellos; que el sector industrial es, ex-ante, competitivo internacionalmente ${ }^{7}$, gracias a lo cual no es necesaria una profunda devaluación para incrementar el crecimiento, lo que podría incluso empeorar las condiciones de competitividad para los sectores ya competitivos internacionalmente (Dvoskin et al, 2018).

\footnotetext{
${ }^{6}$ En el fondo, esta política es la misma que previamente se discutió, es decir, disminución de los salarios.

${ }^{7}$ La postura del neodesarrollismo asume que el sector primario es el sector transable y que el sector industrial es el no transable debido a su falta de competitividad (Bresser, 2008). Sin embargo, esta visión continúa defendiendo el papel de la devaluación, ahora para cerrar las brechas de competitividad entre industrias (Dvoskin y Feldman, 2018).
} 
Es bastante conocido que en las economías de la región la distribución del ingreso tiende a ser una barrera para el crecimiento económico. Lo que estas propuestas sostienen implica un efecto redistributivo del ingreso en favor del capital vía flexibilización laboral y disminución salarial, simultáneamente, con un incremento de la tasa de ganancia. Todo lo cual, reduce la tasa de crecimiento de la demanda efectiva (Amico et al, 2011) y sumerge a las economías de la periferia en profundas recesiones y contracciones económicas.

Estos discursos han significado el abandono de todo proyecto de política industrial y transición tecnológica, pues los diferentes organismos internacionales y los diferentes gobiernos latinoamericanos ${ }^{8}$ han optado por dejar en manos de la autorregulación del mercado la asignación eficiente de los recursos. Esto, amparados en teorías que no son capaces de contemplar la importancia de la mano activa del Estado para determinar transformaciones estructurales y modificar los resultados y las condiciones socioeconómicas de la población.

\section{DESARROLLO DESDE LA DEMANDA: TRES PRINCIPIOS}

Una vez discutidos los principales problemas y las principales fallas teóricas de los discursos y proyectos tradicionales, se hace necesario esbozar una propuesta que busque superar los aparentemente inmutables postulados de la economía del desarrollo tradicional. Así pues, sostenemos, en concordancia con Currie (1968), que la "economía del desarrollo" no existe como campo independiente de la disciplina económica, sino que se trata de la aplicación de la teoría económica a problemas particulares; cuestión que fortalece nuestras posiciones anteriores al defender la necesidad de una coherente y alta teoría (Shackle, 1976). En este orden de ideas, la propuesta que se busca mostrar parte de una concepción de la demanda como la variable clave al momento de pensar cualquier problema de desempeño económico y de una defensa de la política industrial como motor del crecimiento y base fundamental del desarrollo económico.

\section{a. La demanda efectiva}

Este análisis parte de la idea de demanda efectiva propuesta por Keynes (1965), rechazando la ley de Say y planteándola de forma inversa: la demanda crea su propia oferta (Cornwall, 1970). De esta manera, los niveles de empleo y producto son establecidos por los componentes del gasto autónomo, en tanto los niveles de actividad económica determinan las decisiones de inversión de los capitalistas. En otras palabras, es necesario el crecimiento de la demanda para dar pie a la transformación de las condiciones de oferta, esto es, que los niveles esperados de ingreso moldean el empleo y la inversión fijados por los capitalistas (Moreno, 2019).

Entendiendo esto, la inversión privada es impulsada en el corto y largo plazo como una demanda inducida, en lo que se conoce como un "acelerador" de la inversión (Fiorito, 2015). Según Bielschowsky et al. (2013), este efecto es una regularidad para buena parte de las economías latinoamericanas en el período comprendido entre 1950 y 2008, donde se muestra que la inversión está positivamente correlacionada con el crecimiento

\footnotetext{
${ }^{8}$ Son valiosas las excepciones de los gobiernos Kirchneristas en Argentina y de los gobiernos del Partido de los Trabajadores (PT) en Brasil. En los demás gobiernos progresistas de América Latina no han existido mayores propuestas de industrialización o de crecimiento vía demanda efectiva.
} 
económico, sugiriendo que la capacidad productiva se ajusta a las condiciones de la demanda.

En este sentido, el supermultiplicador sraffiano ${ }^{9}$ (Serrano, 1996), que surge como instrumento para solucionar la inestabilidad del modelo de multiplicador de Harrod (1939), vuelve a poner en el centro de las discusiones económicas a la demanda y, en particular, al consumo autónomo, como variable de la demanda que no crea capacidad (como es el caso de la inversión) pero sí lidera el crecimiento económico (Freitas y Serrano, 2007). No obstante, los autores son claros al señalar los límites del crecimiento liderado por la demanda al mostrar que para mantener la estabilidad del sistema es condición suficiente que exista un ajuste parcial o gradual de la tasa de inversión, en otros términos, la tasa de inversión debe expandirse a un ritmo medianamente similar a la tasa de crecimiento de los gastos autónomos, lo que se traduce en que la propensión marginal a invertir debe ser menor a la propensión marginal a ahorrar (Freitas, 2007). En síntesis, el crecimiento es liderado por la demanda y la capacidad productiva que se ajusta a la expansión de los gastos autónomos y de la demanda efectiva (Freitas y Serrano, 2007), adicionalmente, se puede demostrar, de acuerdo con Serrano (1996), que no existe ninguna relación inversa entre los niveles de consumo y de inversión, sugiriendo que no se trata de intereses contrapuestos de política sino, más bien, complementarios.

Precisamente, comprender la importancia de la necesidad de incrementar la tasa de inversión a la par de la tasa de crecimiento del consumo autónomo nos remite al imperativo requerimiento de implementar políticas de demanda atadas a políticas de inversión, en concreto, políticas industriales, pues, como decía Keynes (1965), se deben socializar las inversiones (no los medios de producción) de forma tal que se canalicen los recursos hacia los sectores fundamentales para el crecimiento económico y la ocupación plena, en este caso, la industria, la manufactura, la agricultura y demás sectores claves que requieren de transformaciones tecnológicas y productivas de las que se han visto históricamente excluidos una vez los países desarrollados patean la escalera del progreso tecnológico (Chang, 2002).

\section{b. Desarrollo Industrial}

Kaldor, gran economista húngaro, pensaba que no todos los sectores económicos garantizaban el crecimiento. Sostenía que la industria tiene rendimientos crecientes ${ }^{10}$, asociados a economías de escala y que ayudaba a la acumulación y difusión de conocimiento (Cómbita et al, 2019). Esto lo llevó a formular una serie de leyes del crecimiento económico como un proceso eminentemente endógeno y no exógeno contrario a lo que pretendió mostrar, durante muchos años, la ortodoxia económica, por ejemplo, Solow (1956).

Son tres las leyes de Kaldor: La primera es que existe una fuerte correlación entre el crecimiento del producto manufacturero y el crecimiento del producto. La segunda es

\footnotetext{
${ }^{9} \mathrm{~A}$ este modelo se le llama "sraffiano" o "clásico" ya que asume que la distribución está dada de manera exógena lo que, eventualmente, implica que no existe ningún vínculo necesario entre crecimiento económico y distribución del ingreso (Freitas y Serrano, 2007).

${ }^{10}$ Kaldor seguiría la idea radical de Allyn Young (1928) de ver los rendimientos crecientes como un resultado de la especialización y diversificación de los sectores económicos (Moreno, 2008).
} 
que existe una fuerte relación positiva entre el crecimiento de la productividad industrial y la tasa de crecimiento del producto. La tercera es la relación positiva entre el crecimiento de la productividad de toda la economía y el crecimiento del sector industrial y negativa con el aumento del empleo en los sectores no manufactureros (Moreno, 2008).

Con una muestra de 45 países, Necmi (1999), muestra un fuerte soporte empírico a la posición de que la manufactura es el sector dominante al momento de determinar la tasa de crecimiento del PIB; para el caso de Colombia, Moreno (2008), presenta soportes a la primera ley y Quijano (2019) refuerza la idea de los rendimientos crecientes de la manufactura y de los efectos nocivos de la desindustrialización en los niveles de empleo y producto; para Estados Unidos, McCombie y de Ridder (1983) muestran evidencia empírica que sustenta y fortalece las leyes de Kaldor; igualmente, para un ejercicio más amplio y reciente, Bielschowsky et al. (2013) identifican, como un hecho estilizado, que la productividad tiende a crecer más rápidamente en países con elevadas tasas de crecimiento, sustentando la ley de Kaldor-Verdoorn"11.

\section{c. Finanzas Funcionales}

Ahora, en miras de profundizar y encaminar el análisis hacia propuestas de política económica que permitan a las economías de la periferia superar algunos de sus problemas estructurales, en cuanto al tema del desarrollo se refiere, debemos considerar el papel que juegan las finanzas públicas y los Estados en la formulación de las mismas políticas. De este modo, cuando se proponen políticas industriales, de aumento de salarios o de infraestructura pública, surge entre la tecnocracia y, muchas veces, entre los opinólogos nacionales, una reacción adversa que sostiene que las finanzas del Estado se verán perjudicadas y que los sostenimientos de los eventuales déficits macroeconómicos terminarán por afectar la credibilidad internacional e institucional del mismo modo que afectarán la sostenibilidad financiera de la respectiva nación ${ }^{12}$.

Esta perspectiva comete el grave, y tan común, error tradicional en los hombres prácticos de Keynes de pensar en el Estado como si se tratara de un hogar, convirtiendo las finanzas en un fin en sí mismo y confundiendo la moneda nacional soberana con la moneda extranjera, destruyendo así cualquier pretensión de rigurosidad académica. No obstante, la visión de finanzas funcionales de Lerner (1943 y 1951) se contrapone directamente, y plantea que los déficits y los superávits deben ser instrumentos para obtener el pleno empleo, sin exacerbar la inflación.

Los principios de las finanzas funcionales señalan que no hay límite al endeudamiento del déficit público, en tanto exista desempleo. Sin embargo, esta visión es correcta para el caso de los países del centro global, mientras que, para los países de la periferia, donde existen jerarquías monetarias y donde la restricción de divisas se aparece como el principal problema de cualquier intento de desarrollo, el límite se encuentra en el endeudamiento en moneda extranjera, de la cual el Estado no es emisor y, por ende,

\footnotetext{
${ }^{11}$ Formalizada por Kaldor (1966) y Rowthorn (1975).

${ }^{12}$ Otra de las opiniones muy comunes entre economistas y público en general es que tales políticas generarán inflación por la vía de la demanda, una postura que pierde todo sustento teórico ( $y$, por consiguiente, no es más que ideología) tras los debates del capital (Álvarez et al, 2007). Se sugiere, aquí mismo, que la posición de la inflación como expresión de la pugna distributiva de Sraffa (1960) puede acercarse, más apropiadamente, a contener una explicación concreta y coherente sobre la inflación.
} 
podría entrar en default ${ }^{13}$. Así, los gobiernos de las naciones periféricas deben preocuparse por mantener un nivel estable y alto de reservas y deben procurar, fundamentalmente, controlar tanto los niveles de deuda en moneda extranjera como los intereses pagados por la misma.

Sobre este último punto se hace esencial, como objetivo de cualquier gobierno, que las exportaciones crezcan a un ritmo superior a los intereses de la deuda externa (Pérez y Vernengo, 2020), para garantizar el flujo y la disponibilidad de divisas. Esto significa que se deben reorientar los objetivos de la política fiscal para generar las condiciones de alivio de la restricción externa (Fiorito, 2012), elementalmente, a través de procesos de cambio estructural.

\section{CONCLUSIONES}

Se ha realizado un repaso sobre los fundamentos teóricos de los discursos desarrollistas más populares en América Latina y el Caribe y se ha demostrado por qué no son más que posturas ideológicas producto de una mala y deficiente teoría que, causalmente, sume a las economías de la región en lógicas de especialización productiva en el sector primario donde, agregando los flujos de capitales, se configuran economías basadas en la especulación y en la valorización del capital financiero.

De igual manera, se ha presentado un marco teórico alternativo que ancla el crecimiento económico en el crecimiento de la demanda, yendo en contravía de la economía ortodoxa que se ajusta naturalmente bajo condiciones de oferta. Este nuevo marco teórico permite acercarse, desde un mismo proyecto organizado en diferentes instrumentos de política, al problema del desarrollo en la región, el cual es, fundamentalmente el problema de la restricción externa.

El problema de la disponibilidad de divisas fija límites al crecimiento económico vía demanda efectiva y fija límites a los proyectos de industrialización por sustitución de importaciones, por esta razón se han presentado tres abordajes teóricos que, siendo vitalmente complementarios, pueden inducir un proceso de cambio estructural en las economías de la periferia ${ }^{14}$, estos son; los principios de demanda efectiva, acelerador y supermultiplicador sraffiano, la importancia del sector industrial y las leyes de crecimiento endógeno de Kaldor, y el enfoque de finanzas funcionales para la periferia. Abordajes que, si se articulan en un mismo proyecto de desarrollo nacional (o regional), darían pie a una profunda transformación estructural guiada por la industria y la manufactura que permita superar la configuración desigual centro-periferia y nos permita superar las lógicas especulativas y violentas características de nuestras sociedades.

Adicionalmente, queda el papel de considerar el impacto ecológico y ambiental de estas propuestas. No se busca desconocer la incidencia de la industria y la manufactura en la producción y distribución de diferentes gases efecto invernadero y de contaminación material de las fuentes hídricas. En este sentido, sostenemos que es fundamental reconocer la importancia de transiciones energéticas y productivas hacia industrias bajas

\footnotetext{
${ }^{13}$ La salida del default puede significar medidas de austeridad y de ajuste neoliberal, razón por la cual es importante garantizar la sostenibilidad de la deuda externa si se quiere mantener el espacio fiscal de cualquier país.

${ }^{14}$ Es decir, disminuir la elasticidad ingreso de las importaciones e incrementar la elasticidad ingreso de las exportaciones.
} 
en carbono y energías no contaminantes, que den pie a un crecimiento económico no sólo sustentable sino ambientalmente sostenible.

Buena parte de estas reflexiones se encuentran atadas a un retomar de los economistas clásicos, grandes pensadores que tenían un interés científico auténtico por entender la sociedad capitalista y su forma de coordinación, estos filósofos terrenales (Heilbroner, 2015) se preguntaron siempre por las condiciones de reproducción de la sociedad, y respondieron esbozando una (y múltiples) teoría del valor como eje fundamental para garantizar la armonía y el "equilibrio" de una organización social de agentes descentralizados.

Todo esto deja en entredicho a la disciplina económica y a su enseñanza y reproducción a nivel académico, político y social. El paradigma dominante en economía ha venido perpetuándose gracias a los interese políticos detrás del mismo, de otra forma, ¿cómo se explica que habiendo tantos - y tan grandes - economistas neoclásicos que han aceptado las críticas de Cambridge se siga enseñando la misma función de producción agregada en todos los cursos de fundamentos de economía? Finalmente, se hace necesario retomar la teoría económica y retomar las grandes preguntas (como las de los economistas clásicos), pues, en los últimos años, se ha abandonado todo interés teórico y los economistas se han enfocado en refinar sus técnicas econométricas y matemáticas en búsqueda de acercarse, casi exclusivamente, a contenidos empíricos buscando presentar la realidad como un dato y pretendiendo negar, implícitamente, que se encuentran presos de economistas difuntos.

La reflexión final invita a desnaturalizar el marco institucional y las configuraciones político-económicas del modo de producción, entendiendo que, si se quiere optar por un modelo de desarrollo anclado en las políticas industriales y de crecimiento vía demanda, es una condición necesaria desmontar los aparatos neoclásicos de regulación (o desregulación) ${ }^{15}$ que impiden transformaciones en el núcleo de las relaciones sociales de producción. En el fondo, se aboga por un idealismo filosófico, por una filosofía de la acción (Fusaro, 2018).

\section{REFERENCIAS}

Alvarez, E., Dvoskin, A., Feldman, G. y Libman, E. (2007). Sobre la relación entre la controversia de Cambridge y la inflación por exceso de demanda. I Jornadas de Economía Política. Instituto de Industria. Universidad Nacional de General Sarmiento.

Amico, F., Fiorito, A. y Hang, G. (2011). Producto potencial y demanda en el largo plazo: hechos estilizados y reflexiones sobre el caso argentino reciente. Centro de economía y finanzas para el desarrollo de la Argentina (CEFIDAR). Documento de trabajo, No. 35.

Austin, J. (2016). Cómo hacer cosas con palabras. Barcelona: Paidós.

Bielschowsky, R. (2009). Sesenta años de la CEPAL: estructuralismo y neoestructuralismo. Revista CEPAL, 97, pp. 173-194.

\footnotetext{
${ }^{15}$ Vale la pena hacer dos precisiones finales: 1) Neoclasicismo no es neoliberalismo, el neoclasicismo es una corriente teórica, el neoliberalismo es una ideología de derechas, y 2) Usualmente, se asocia al neoliberalismo con una disminución del aparato estatal, cuando en realidad se trata de un activo intervencionismo del mismo, tanto para establecer las instituciones necesarias para el libre desarrollo de la idea del mercado autorregulado como para garantizar su perpetuación y reproducción en el tiempo (Polanyi, 2017).
} 
Bielschowsky, R., Schönerwald, C. y Vernengo, M. (2013). Padrões de desenvolvimento económico (1950-2008): América Latina, Ásia e Rússia Vol I y II. Brasília: Centro de Gestão e Estudos Estratégicos, Cap 1, pp. 21-78.

Blaug, M. (1985). Economic theory in retrospect. Cambridge University Press.

Bonizzi, B. (2013). Financialization in developing and emerging countries. International Journal of Political Economy, 42 (4), pp. 83-107.

Bortz, P. y Kaltenbrunner, A. (2018). The international dimension of financialization in developing and emerging economies. Development and change, 49 (2), pp. 375-393.

Bresser-Pereira, L. (2008). The Dutch disease and its neturalization: a Ricardian approach. Brazilian Journal of Political Economy, 28(1), pp. 47-71.

Chang, H-J. (2002). Kicking away the ladder - Development strategy in historical perspective. Londres: Anthem Press.

Combita, G., Mora, A. y Moreno, A. (2019). Política industrial moderna: fundamentos e importancia para el crecimiento económico y la igualdad. Universidad Nacional de Colombia. Facultad de Ciencias Económicas, Documento FCE-CID No. 100. Escuela de economía.

Comisión Económica para América Latina y el Caribe (CEPAL). (2012). Cambio estructural para la igualdad. Una visión integrada del desarrollo. Sesiones de la CEPAL, No. 34, San Salvador.

Cornwall, J. (1970). The role of demand and investment in long-term growth. Quarterly Journal of Economics, vol. 84, no. 1, 48-69.

Crespo, E., Dvoskin, A. e lanni, G. (2019). Exclusion in "Ricardian" trade models. Centro Sraffa. Working Paper, No. 39.

Currie, L. (1968). Desarrollo económico acelerado. La necesidad y los medios. México: Fondo de Cultura Económica.

Dornbusch, R., Fischer, S. y Samuelson, P. (1977). Comparative advantages, trade and payments in ricardian model with a continuum of goods. American Economic Review, 67 (5), pp. 823-839.

Dvoskin, A. y Feldman, G. (2018). A formal assessment of new-developmentalist theory and policy, Brazilian Journal of Political Economy, 38 (3), pp. 395-413.

Dvoskin, A., Feldman, G. y lanni, G. (2018). New-Structuralist exchange-rate policy and the pattern of specialization in Latin American countries. Centro Sraffa. Working Paper, No. 28.

Fiorito, A. (2012). Finanzas funcionales en economías abiertas. Estudios Latinoamericanos, Nueva Época, 30.

Fiorito, A. (2015). Un enfoque clásico-keynesiano de los precios, la distribución del ingreso y el crecimiento en Argentina. Revista del Departamento de Ciencias Sociales, 2 (4), pp. 83-102.

Frenkel, R. y Ros, J. (2006). Unemployment and the real Exchange rate in Latin America. World Development, 34 (4), pp. 631-646.

Freitas, F. (2007) Notes on the dynamic stability of the flexible supermultiplier demand led growth model. Mimeo, IE-UFRJ.

Freitas, F. y Serrano, F. (2007). El supermultiplicador sraffiano y el papel de la demanda efectiva en los modelos de crecimiento. Revista Circus, 1, pp. 21-37, Ciudad de Buenos Aires.

Fusaro, D. (2018). Idealismo o barbarie, Madrid: Trotta.

Garegnani, P. (1978). Notes on consumption, investment and effective demand: I. Cambridge Journal of Economics, 2, 253-271. 
Garegnani, P. (1979). Notes on consumption, investment and effective demand: II. Cambridge Journal of Economics, 3, 63-82

Harrod, R. (1939). La teoría dinámica. En A. Sen, Economía del crecimiento, México: Fondo de Cultura Económica, 1979.

Hayek, F. (1946). La teoría pura del capital. Editorial Aguilar.

Heilbroner, R. (2015). Los filósofos terrenales. Madrid: Alianza Editorial.

Kaldor, N. (1957). A model of economic growth. Economic Journal, Vol. 67, No. 268, pp. 591-624.

Kaldor, N. (1966). Causes of the slow rate of economic growth in the United Kingdom. The Essential Kaldor. Duckworth.

Kaldor, N. (1972). The irrelevance of equilibrium economics. Economic Journal, Vol. 82, No. 328, pp. 1237-1255.

Kaldor, N. (1975). What is wrong with economic theory. The Quaterly Journal of Economics, 89 (3), pp. 347-357.

Keynes, J. (1965). Teoría general del interés, la ocupación y el dinero. México: Fondo de Cultura Económica.

Krugman, P. y Taylor, L. (1978). Contractionary effects of devaluation. Journal of International Economics, 8, pp. 445-456.

Krugman, P. (1991). Myths and realities of US competitiveness. Science, 254 (5033), pp. 811.815.

Lerner, A. (1943). Functional finance and the federal debt. Social Research.

Lerner, A. (1951). Economics of employment. New York: McGraw-Hill.

McCombie, J., de Ridder, J. (1983). Increasing returns, productivity and output growth: the case of the United States. Journal of Post Keynesian Economics, 5, pp. 373-387.

Moreno, A. (2008). Las leyes del desarrollo económico endógeno de Kaldor: El caso colombiano. Revista de Economía Institucional, 10 (18), pp. 129-147.

Moreno, A. (2019). El principio de la demanda efectiva. ¡La demanda efectiva es un punto! Revista Papeles, 11 (21), pp. 55-66.

Necmi, S. (1999). Kaldor's growth analysis revisited. Applied Economics, 31 (5), pp. 653-660.

Parrinello, S. (1979). Distribution, growth and international trade. Cambridge: Harvard University Press.

Polanyi, K. (2017). La gran transformación: los orígenes políticos y económicos de nuestro tiempo. México: Fondo de Cultura Económica.

Prebisch, R. (1983). Obras escogidas. Colombia: Plaza y Janes.

Rapetti, M., Razmi, A. y Skott, P. (2012). The real exchange rate and economic development. Structural change and economic dynamics, 23 (2), pp. 151-169.

Rowthorn, R. (1975). A Reply to Lord Kaldor's Comment. The Economic Journal 85, 340, pp. 897901.

Samuelson, P. (1966). A summing up. The Quarterly Journal of Economics, 80 (4).

Serrano, F. (1996). The Sraffian Supermultiplier. Unpublished Ph.D. Dissertation, Cambridge University, Cambridge. 
Shackle, G. (1967). The years of high theory. Invention and Tradition in economic thought. Cambridge: Cambridge University Press.

Solow, R. (1956). A contribution to the theory of economic growth. Oxford: Quarterly Journal of Economics, 70, pp. 65-49.

Solow, R. (1975). Brief comments. The Quarterly Journal of Economics, 89 (1), 48-52.

Sraffa, P. (1960). Producción de mercancías por medio de mercancías. Preludio a una crítica de la teoría económica. Barcelona: Oikos.

Thirlwall, A. (1979). The Balance of Payments Constraint as an Explanation of International Growth Rate Differences. Banca Nazionale del Lavoro Quarterly Review, 32 (128), 45-55.

Young, A. (1928). Rendimientos crecientes y progreso económico. En K, Arrow y T, Scitovsky, comps. La economía del bienestar, Vol I, México: Fondo de Cultura Económica. 